\title{
Face Recognition Framework based on Convolution Neural Network with modified Long Short Term memory Method
}

\author{
1Sushmitha Parikibanda \\ ${ }^{1}$ National College of Ireland, Dublin, Ireland. \\ ${ }^{* *}$ Corresponding Author: sushmithaparikibanda@gmail.com
}

Received: 20.08 .2020 ,

Revised: 14.11 .2020

Accepted: 16.12 .2020 ,

Published: 22.12.2020

DOI:

10.53409/mnaa.jcsit20201304

Abstract: For real-world applications, such as video monitoring, interaction between human machines and safety systems, face recognition is very critical. Deep learning approaches have demonstrated better results in terms of precision and processing speed in image recognition compared to conventional methods. In comparison to traditional methods. While facial detection problems with different commercial applications have been extensively studied for several decades, they still face problems with many specific scenarios, due to various problems such as severe facial occlusions, very low resolutions, intense lighting and exceptional changes in image or video compression artifacts, etc. The aim of this work is to robustly solve the issues listed above with a facial detection approach called Convolution Neural Network with Long short-term Model (CNNmLSTM). This method first flattened the original frame, calculating the gradient image with Gaussian filter. The edge detection algorithm Canny-Kirsch Method will then be used to identify edge of the human face. The experimental findings suggest that the technique proposed exceeds the current modern methods of face detection.

Keywords: Brain tumor, Computer-Aided Diagnosis, Deep Learning, Fusion Feature, Recurrent Extreme Learning Machine, Artificial Bee Colony.

\section{INTRODUCTION}

ace recognition is the mechanism by which the vision system identifies a 1 particular person's face. Due to its use in surveillance systems, access control, video monitoring, business areas and also in social networks such as Facebook it was a key tool for human-computer interaction. Face recognition has once again gained attention since the rapid growth of artificial intelligence due to its non-intrusive nature and because it is the predominant way to recognize individuals by contrasting them with other forms of biometric techniques. Without the awareness of the subject individual in an unregulated setting, face recognition can be easily verified. When investigating the history of face recognition, several academic papers have studied it[1]. Traditional approaches focused on superficial learning face challenges such as pose variation, facets, scene lighting, image context ambiguity, and changes in facial expression as in references [2]. Shallow learning methods use only certain basic image features to extract sample functions and rely on artificial experience.

Deep learning will extract more complex facial characteristics [3]. Deep learning makes significant strides in solving problems that have for many years been limiting the best efforts of the artificial intelligence community. It has proven excellent by exposing high-dimensional data tough structures and thus applies in many fields of science, industry and government. It tackles the question of learning hierarchical representation with one or more algorithms and has predominantly broken records in the fields of image recognition, natural language processing, semantic segmentation and a variety of other scenarios in the real world [5].

Deep learning methods like $\mathrm{CNN}$, the Stacked Autoencoder and the Deep Belief Network (DBN) are distinct. In image recognition and facial recognition, $\mathrm{CNN}$ is also used. $\mathrm{CNN}$ is a type of artificial neural networks which use the convergence methodology to extract the input data features to increase features. Furthermore, the use of computer vision approaches has increased through CNN's use in the resolution of 
many other computer vision activities, such as object detection and identification, segmentation, optical character recognition, facial expression analyzes, age estimates, and so on.

The effect of that is to reduce the memory needs and to reduce correspondingly the number of parameters to be learned. This increases the efficiency of the algorithm. At the same time, the images must be preprocessed or derived from other computer algorithms. Such operations, however, are rarely required for $\mathrm{CNN}$ processing images. It's another algorithm that can't learn by computer. In-depth research still exists several limitations. For this purpose, Convolutional Neural Network with Long Short Term (CNNmLSTM) changed memory was proposed. The original image was initially smoothed with a Gaussian filter during the preprocessation step, and its gradient value was measured. The edge detection algorithm Canny-Kirsch Method is then used to detect edge of the human face. After this function is extracted using a self-Residual Attention-based Network (SRANet), the device collects the global dependencies of spatial and channel dimensions for discriminatory facial feature integration.

The structuring of the rest of paper is as follows: In Section 2, discuss existing face recognition methods. Section 3 describes the proposed methodology. Section 4 describes the results and discussion; in Section 5, conclude the paper and define the future work.

\section{RELATED WORK}

An inherent correlation between detection and augmentation is proposed under the deep cascading multifunction system in [6] to improve their performance. We exploit a cascaded architecture with three stages in which deep convolutional neural networks are deliberately created to roughly forecast the face and landmark.

A CNN cascade multi-task system [7] that contains both tasks. We show that multi-task face recognition learning and the head pose estimation helps obtain more representative characteristics.

For face detection, [8] is proposed based on skin color segmentation and facial properties. An Algorithm is a competent analytical tool to evaluate different color patterns such as RGB, YcbCr, and HSV, as well as their skin color detection combinations.

In [9] a new approach called the algorithm DPAdaboost proposed for the detection of the human face and the improvement of the right rate of detection. The multi-angle face is identified by an enhanced Adaboost algorithm with the combination of a frontal face grader and a profile face grader.

The architecture in cascading with three phases carefully designed [10] to forecast the presence of faces, based on deep convolutional networks. In [11] an important facial recognition system has been developed to index a specific face from various video images. The classifier used is the enhanced neural grid that optimizes weight factors with the modified cuckoo search algorithm.

\section{PROPOSED METHODOLOGY}

This research proposes a facial recognition system with modified Long Short Term (CNNmLSTM) memory from the Convolutional Neuronal Network. The architectural structure is of three layers and recognizes all picture regions containing images. The face detection is an automated face reconnaissance device pre-processing level. In the first step, Retinex adaptive filter is used for improving the image to eliminate unnecessary noise. At the next level, a Canny-Kirsch Method edge detection is performed on the face edge and functional extraction takes place using SCA and SSA. The CNN-mLSTM then classifies the unknown class or class that is not face to face as either face to face or non-face. The block diagram of the proposed CNN-mLSTM based face recognition is illustrated in Figure 1.

\subsection{Input database}

The database was established in February 2002 at the IIT Kanpur campus. There are forty subjects, and 11 different images per subject. Few more photos are included for certain subjects. All pictures are brightly homogeneous at the backdrop. The subjects are frontally straight. The pictures had been in JPEG format. Each image has a resolution of $640 \times 480$, with 256 gray per pixel. The male and female images have been put in two principal folders. Every subject has eleven distinct images in both folders. Database are variations based on orientation and emotion. 


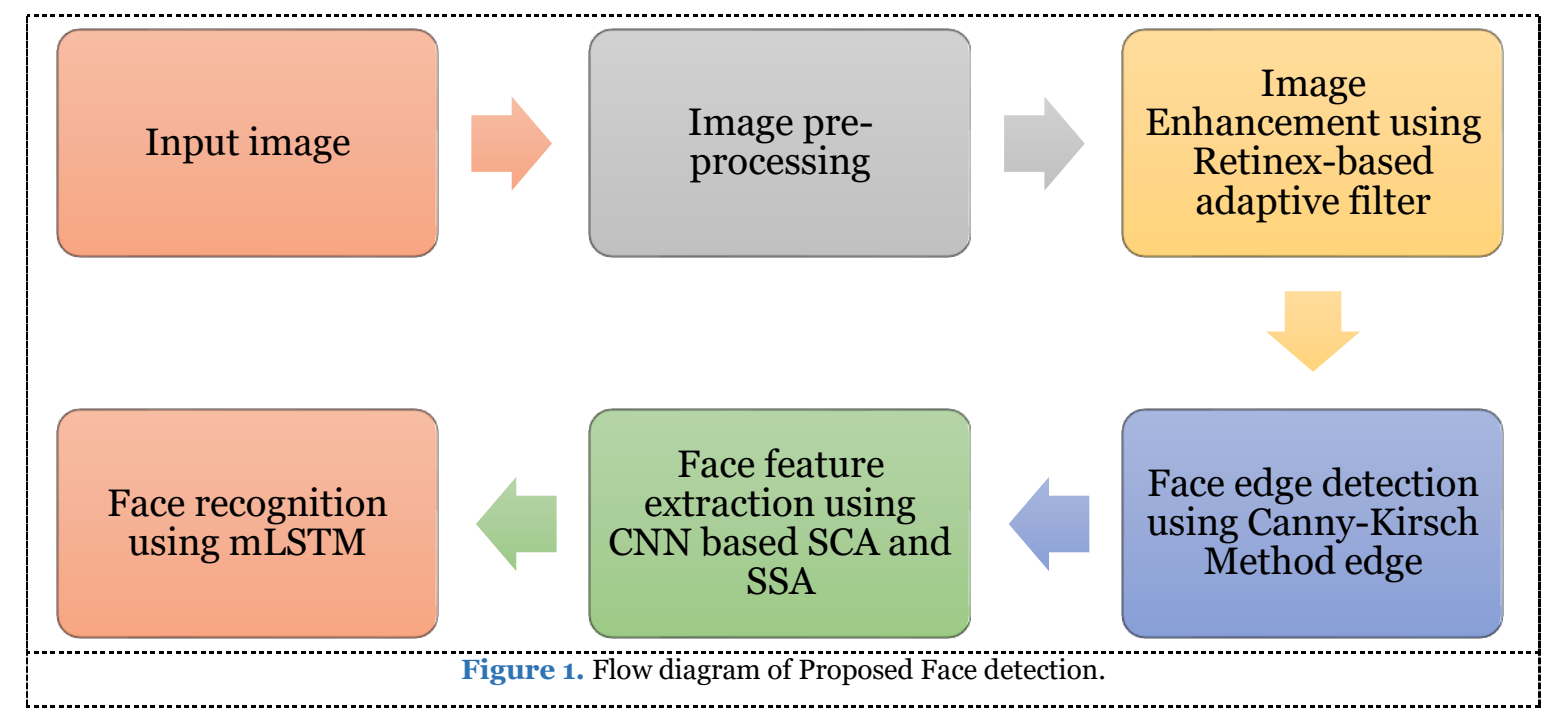

\subsection{Image Pre-processing Step}

In pre-processing of images, since the various conditions, such as the different sources, can affect the final result. The proposed system would change the facial expression luminous intensity to be registered in the picture files. Higher pixel interval brightness to calculate the mean in this image, and used this average value as a reference and calculation in equation ( 1 \& 2);

$$
\begin{gathered}
R_{a}=\frac{\sum_{1}^{n} \max N_{R}}{n}, G_{a}=\frac{\sum_{1}^{n} \max N_{G}}{n}, B_{a} \\
=\frac{\sum_{1}^{n} \max N_{B}}{n} \\
R^{\prime}=\frac{255}{R_{a}} * N_{R}, G^{\prime} \\
=\frac{255}{G_{a}} * N_{G}, B^{\prime} \\
=\frac{255}{B_{a}} * N_{B}
\end{gathered}
$$

Adjustments of the original picture pixel based on mean values. Where $N_{R}, N_{G}$ and $N_{B}$ represent pixel values of the original image. In the color channel interval of $n, R, G$ and $B$ represent the average value of a pixel, and here $n$ represent the total number of pixels to obtain a brightness range, and represent pixel values after the configuration. This pre-processing image is used to change the low light images.

\subsection{Image Enhancement using Retinex- based adaptive filter}

Retinex based adaptive filter is a proposed framework for enhancing color images in this segment. This system can be used to improve conventional 24-bit images as well as to compress high dynamic range images generated from raw format or multiple exposure techniques that are linear RGB images. Let Retinex theory describe the portion of luminance treated as:

$$
\begin{aligned}
& \operatorname{Retinex}_{Y} \\
& =\log _{10}\left(I_{Y}^{\prime}\right)-\log _{10}(\text { mask })
\end{aligned}
$$

Where $\log _{10}\left(I_{Y}^{\prime}\right)$ is the $Y$ portion of the non-linear RGB image I and translated to the color space of YcbCr. The last term mask, is a matrix representing the weighted average of its surrounding for each pixel. A significant factor is how you describe this surrounding and its corresponding weights. A traditional approach is to define the mask with a filter conveying the color.

$$
\text { mask }=I_{Y}^{\prime} * \mathrm{LPF}
$$

Where LPF is a circularly symmetric low-pass filter that is strictly defined by a 1-dimensional function rotated around the $\mathrm{z}$ axis and the 1dimensional curve is typically defined by a simple Gauussian or Gauussian function composition. The 1-dimensional radial function is a Gaussian curve, whose spatial constant varies depending on the local contrast of the face image. The spatial constant is given by equation (5) with an initial value $\ddot{y}$. If a strongly contrasted edge is marked along the radius, there will be 8 divided into $\sigma$. 


$$
\begin{aligned}
\sigma=\frac{r_{\max }}{8}, \text { where } & \max \left(I_{\text {size }}\right) \\
= & r_{\max }
\end{aligned}
$$

Although the weights and support of the filter are modified for each pixel, the mask is determined sequentially pixel after pixel and $\operatorname{mask}(\mathrm{x}, \mathrm{y})$ is the weighted sum of elements in the Coordinate $(x, y)$ pixel surround.

$$
\begin{aligned}
& \operatorname{mask}(x, y) \\
& =\int_{\theta=0}^{360} \int_{r=0}^{r_{\max }} I_{Y}(x+\cos \theta), y \\
& +\sin (\theta) e^{-\frac{r^{2}}{\sigma^{2}}}
\end{aligned}
$$

Where $\sigma$ is the Gaussian spatial constant which varies in the direction of radius. In this way, the support of the filter follows essentially the high contrast facial edges of the image. Using the Kirsch edge detector these face edges are detected.

\subsection{Face Feature Extraction and recognition using $C N N$}

A face recognition method based on CNN-CECLSTM is proposed in this section. And the network used here consists of nine layers. These layers contains convolution layers, pooling layers, fullconnected layers and Softmax regression layer. The convolution layers and the pooling layers are used for feature extraction followed by fullconnected layers, and the last layer uses a CECLSTM classifier with strong non-linear classification capability. The CNN based feature extraction is illustrated in Figure 4.

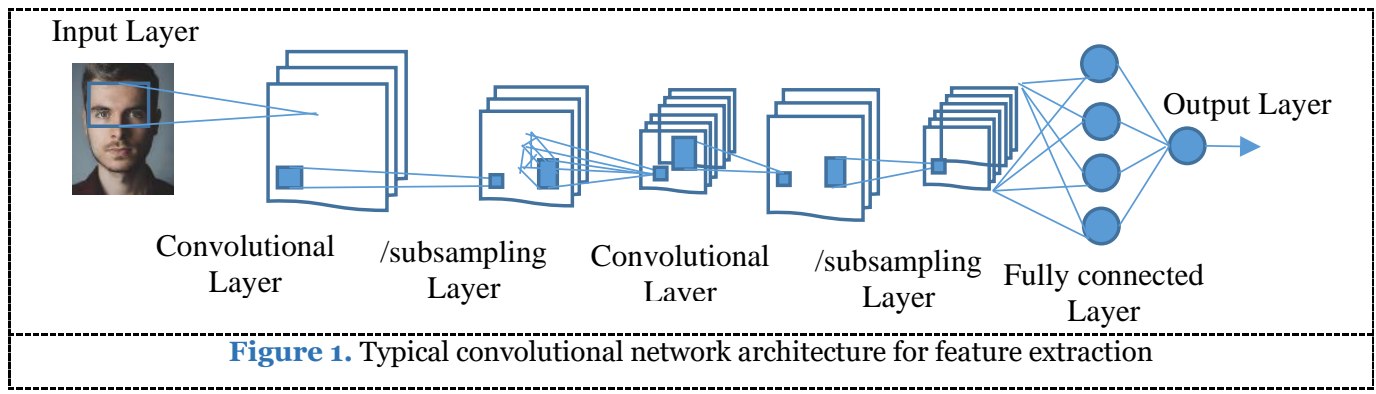

$\mathrm{CNN}$ comprises three types of layers: input, convolution, and pooling, and layers which are fully connected. In the input layer, the obtained information consists of multiple image sequences $\left\{I_{1}, I_{2}, \ldots, I_{n}\right\}$, which consist of the dataset indicator diagram. Built on the original $\mathrm{CNN}$ architectures, add attention modules to each of the ResNet structure's residual bottlenecks to get a refined face function. In particular, the proposed attention module consists of two blocks called the selfresidual channel attention module and the selfresidual spatial attention module, which sequentially learns the channel relationship matrix and spatial relationship matrix, and then achieves the refined function by multiplication of matrixes.

For instance, assumed an intermediate feature map FM, the channel refined feature FC and the spatially refined feature FS can be sequentially obtained. Moreover, it argues that the features derived from the global average pooling layer are not adequately inclusive for deep face recognition, so instead, use a completely linked layer. With the above modifications, the information redundancy between channels can be reduced as well as the most important part of face images can be learnt. At last, the refined function can be obtained by residual shortcut learning. The function vectors received will then be fed to the sequential sheet. To capture long distance dependency, mLSTM for vector composition is inserted into the sequential layer.

\subsection{Modified Long Short Term Memory (mLSTM) for Face Recognition}

Recurrent Neural Network (RNN) is an enhanced version of LSTM. Instead of traditional simple RNN modules, LSTM implements memory blocks to tackle the issue of the gradient vanishing and bursting. LSTMs are also better able to manage long term dependencies than conventional RNNs. This means that LSTMs can recall and relate past knowledge to the present (which actually lags very far back in time as opposed to the present). A memory block in LSTM is a complex processing unit, which consists of one or more memory cells. A pair of multiplicative gates are being used as gateway for input and output. A collection of adaptive, multiplicative gates regulates the full operations of a memory block. The output of the input gate makes or discards activity for the input flow to a memory cell from a cell activation.

The efficiency of the output gate makes or discards operation to other nodes for an output state of a memory cell. Forgetting gate and peephole connections were integrated into the current LSTM network as research on LSTM progressed. Instead of the Persistent error carousel (CEC), the Forget 
gate is used. The forget gate allows to forget or reset a memory cell's states. The peephole connections are made to all of its gates from a memory cell. They study both the exact timing of the outputs and the internal state of a memory cell. The mLSTM function is as follows.

CNN's features input sequence is fed into the mLSTM architecture. In the recurrent hidden layer (h) of LSTM architecture, the output sequence of continuous write, read, and reset operations by three multiplicative units (input (i), output (o), and forget gate (f)) on the memory cell (c) is calculated iteratively from $j=1,2, \ldots, j+1$. The sequence of operations taking place in mLSTMs at time step $j$ can be fleetingly signified by the below equation (7).

$$
\begin{gathered}
i_{t}=\sigma\left(w_{x i} x_{j}+w_{h i} h_{j-1}+w_{c i} c_{j-1}+b_{i}\right) \\
f_{j}=\sigma\left(w_{x f} x_{j}+w_{c f} h_{j-1}+w_{c f} c_{j-1}+b_{f}\right) \\
c_{j}=f_{j} \odot c_{j-1}+i_{j} \odot \tan h\left(w_{x c} x_{j}\right. \\
\left.+w_{h c} h_{j-1}+b_{c}\right) \\
o_{j}=\sigma\left(w_{x o} x_{j}+w_{c o} h_{j-1}+w_{c o} c_{j-1}+b_{o}\right) \\
h_{j}=o_{j} \odot \tanh \left(c_{j}\right) \\
y_{j}=w_{y h} h_{j}+b_{y}
\end{gathered}
$$

Where $\odot$ remains the scalar product of two vectors and $\sigma()$ means the standard logistics sigmoid function defined as follows (9)

$$
\sigma(x)=\frac{1}{1+e^{-x}}
$$

At this time weight matrices denoted as $w$ and bias vectors $b$ are used to build connections bet ween the input layer, output layer and memory block. CNN in this CNN-mLSTM consists only of layer convolution and layer maxpooling. The maxpooling layer output is transmitted to the subsequent LSTM layer.

$$
y_{j}=\operatorname{CNN}\left(x_{i}\right)
$$

Here $x_{i}$ remains the initial input vector to the $\mathrm{CNN}$ network with the class label and $y_{j}$ stands the output of the CNN network to be nourished to the next mLSTM network $x_{i}$ the feature vector fashioned from the max-pooling operation in CNN. Learning about the long-range temporal dependencies is fed to the MLSTM.

\section{EXPERIMENTAL RESULTS AND DISCUSSION}

The performance of the proposed $\mathrm{CNN}$ mLSTM is evaluated in this section, and the performance results are compared with existing CNN [7], DP-Adaboost [9] and improved neural network [11] face detection schemes. The facts given below show that the device proposed has achieved better performance in terms of precision, fmeasurement, recall and accuracy.

Precision: It reflects the proportion of positive samples correctly classified as expected in equation (6):

$$
\text { Precision }=\frac{T P}{F P+T P}
$$

Recall: The recall of a classifier reflects the positive samples properly assigned to the total number of positive samples and is calculated as in equation (12):

$$
\text { Recall }=\frac{T P}{T P+F N}
$$

F-measure: this is also referred to as F 1-score, and as in equation (13) is the harmonic mean of precision and recall:

$$
F-\text { measure }=\frac{2 *(\text { Recall } * \text { Precision })}{(\text { Recall }+ \text { Precision })}
$$

Accuracy: This is one of the most frequently used performance classification measures and is defined as a ratio between the correctly classified samples and the total number of samples as in equation (14):

$$
\text { Accuracy }=\frac{T P+T N}{T P+T N+F P+F N}
$$

Where true positive (TP) samples are properly classified faces, false positive (FP) samples are incorrectly classified correctly, True negative (TN) samples are not properly classified, and false negatives (FN) are incorrectly classified faces.

\subsection{Precision Rate comparison}

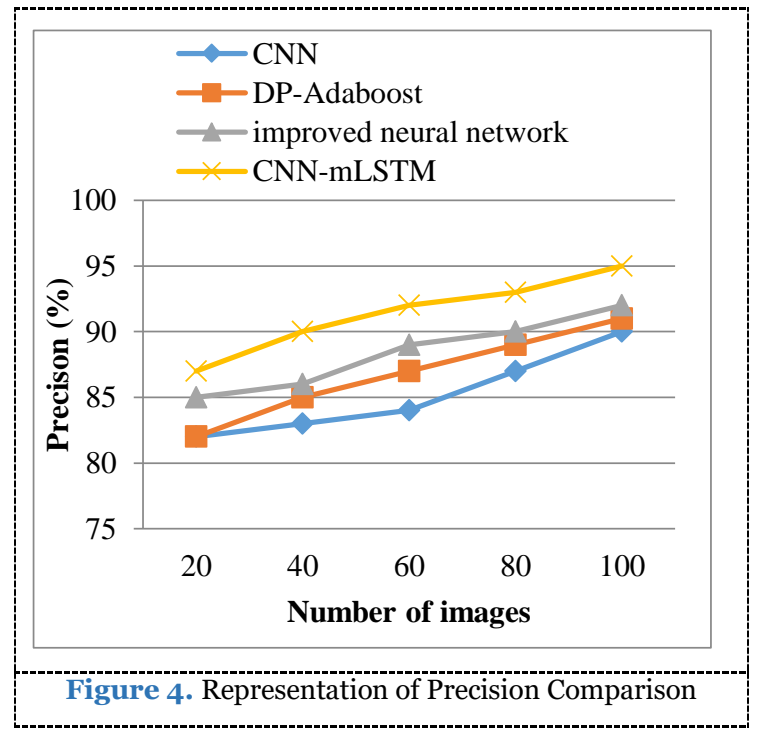

From the above Figure 4, the graph shows how accurate the number of images in the specified datasets is compared. These methods are implemented as CNN, DP-Adaboost, improved neural network and CNN-mLSTM. When the number of records increases according to the precision value. From this graph, it is learned that 
the proposed CNN-mLSTM offers 95\% higher precision than previous methods that yield better results in the detection of face. The numerical results of Precision Comparison is shown in Table 1.

Table 1. The numerical results of Precision Comparison

\begin{tabular}{ccccc}
\hline $\begin{array}{c}\text { No.of } \\
\text { image } \\
\mathbf{S}\end{array}$ & $\begin{array}{c}\mathbf{C N} \\
\mathbf{N}\end{array}$ & $\begin{array}{c}\text { DP- } \\
\text { Adaboos } \\
\mathbf{t}\end{array}$ & $\begin{array}{c}\text { improve } \\
\text { d neural } \\
\text { network }\end{array}$ & $\begin{array}{c}\text { CNN- } \\
\text { mLST } \\
\mathbf{M}\end{array}$ \\
\hline 20 & 82 & 82 & 85 & 87 \\
\hline 40 & 83 & 85 & 86 & 90 \\
\hline 60 & 84 & 87 & 89 & 92 \\
\hline 80 & 87 & 89 & 90 & 93 \\
\hline 100 & 90 & 91 & 92 & 95 \\
\hline
\end{tabular}

\subsection{Recall comparison}

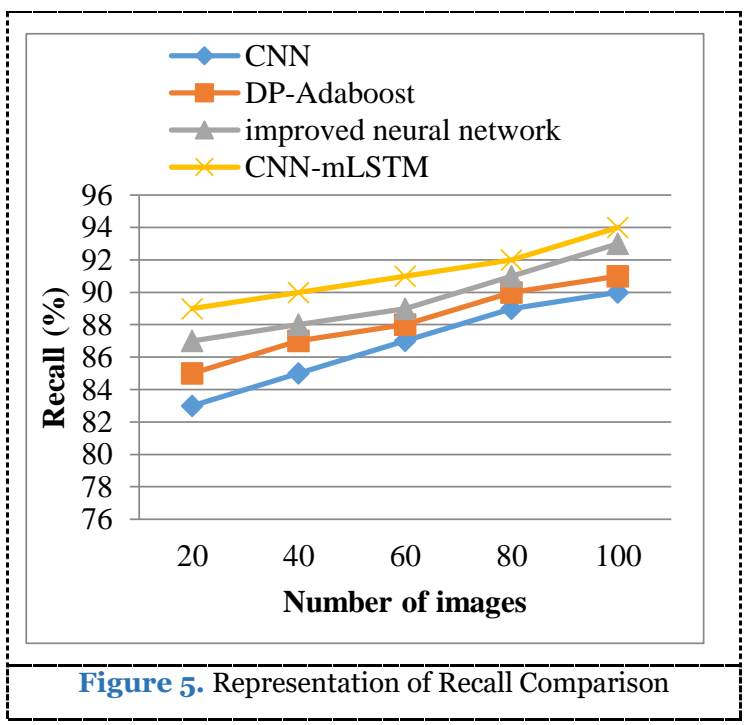

From the above Figure 5 the graph illustrates the recall relation for the number of images in the listed datasets. These methods are implemented as CNN, DP-Adaboost, improved neural network and $\mathrm{CNN}$ mLSTM. Increasing the number of images often increases the correct value for the recall. Through this graph, it is discovered that the current CNNmLSTM offers recall $94 \%$ higher than previous methods. The explanation for this is that the CNNmLSTMextracts the features directly which will enhance the face detection results. The numerical results of Recall Comparison is shown in Table 2.

Table 2. The numerical results of Recall Comparison

\begin{tabular}{ccccc}
\hline $\begin{array}{c}\text { No.of } \\
\text { image } \\
\text { S }\end{array}$ & $\begin{array}{c}\text { CN } \\
\text { N }\end{array}$ & $\begin{array}{c}\text { DP- } \\
\text { Adaboos } \\
\text { t }\end{array}$ & $\begin{array}{c}\text { improve } \\
\text { d neural } \\
\text { network }\end{array}$ & $\begin{array}{c}\text { CNN- } \\
\text { mLST } \\
\text { M }\end{array}$ \\
\hline 20 & 83 & 85 & 87 & 89 \\
\hline 40 & 85 & 87 & 88 & 90 \\
\hline 60 & 87 & 88 & 89 & 91 \\
\hline 80 & 89 & 90 & 91 & 92 \\
\hline 100 & 90 & 91 & 93 & 94 \\
\hline
\end{tabular}

\subsection{F-measure Rate comparison}

Sushmitha Parikibanda. Face Recognition Framework. 2020

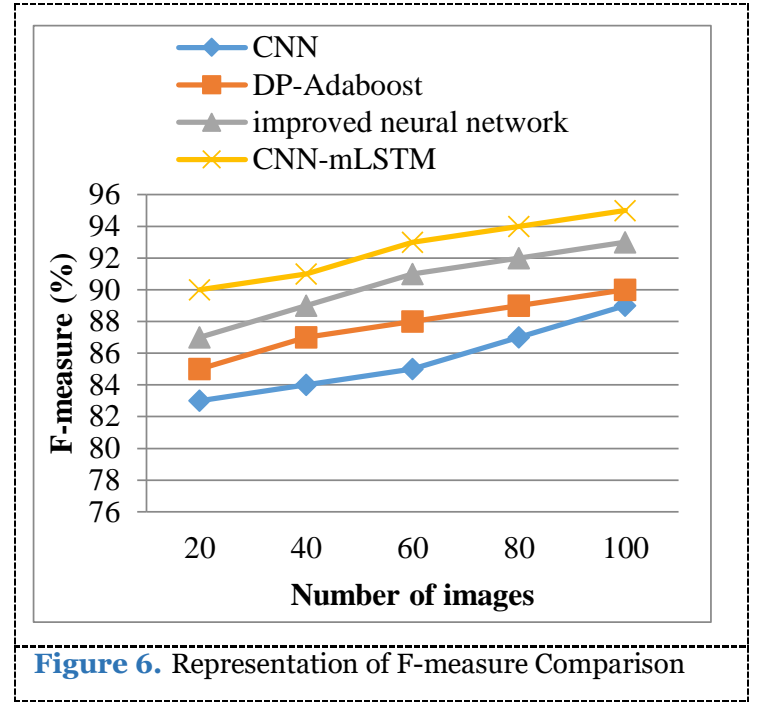

From the above Figure 6, the graph explains the fmeasure relation for the number of images in the given datasets. These methods are implemented as CNN, DP-Adaboost, improved neural network and CNN-mLSTM. When the number of data is increased, and the f-measure value is increased accordingly. From this graph it is learned that the proposed CNN-mLSTM offers 95\% higher fmeasure than previous methods. Therefore the proposed CNN-mLSTM algorithm is stronger than the current algorithms in terms of better performance of classifying face. The numerical results of F-measure Comparison is shown in Table 3.

Table 3. The numerical results of F-measure Comparison

\begin{tabular}{ccccc}
\hline $\begin{array}{c}\text { No.of } \\
\text { image } \\
\mathbf{S}\end{array}$ & $\begin{array}{c}\mathbf{C N} \\
\mathbf{N}\end{array}$ & $\begin{array}{c}\text { DP- } \\
\text { Adaboos } \\
\mathbf{t}\end{array}$ & $\begin{array}{c}\text { improve } \\
\text { d neural } \\
\text { network }\end{array}$ & $\begin{array}{c}\text { CNN- } \\
\text { mLST } \\
\mathbf{M}\end{array}$ \\
\hline 20 & 83 & 85 & 87 & 90 \\
\hline 40 & 84 & 87 & 89 & 91 \\
\hline 60 & 85 & 88 & 91 & 93 \\
\hline 80 & 87 & 89 & 92 & 94 \\
\hline 100 & 89 & 90 & 93 & 95 \\
\hline
\end{tabular}

\subsection{Accuracy comparison}

From the above Figure 7 the diagram illustrates the processing time relation for the number of images in the specified datasets. These methods are implemented as CNN, DP-Adaboost, improved neural network and CNN-mLSTM. From this graph it is known that the proposed CNN-mLSTM algorithm is higher than the existing algorithms with a high precision rate of $96 \%$ in terms of better template matching results. 


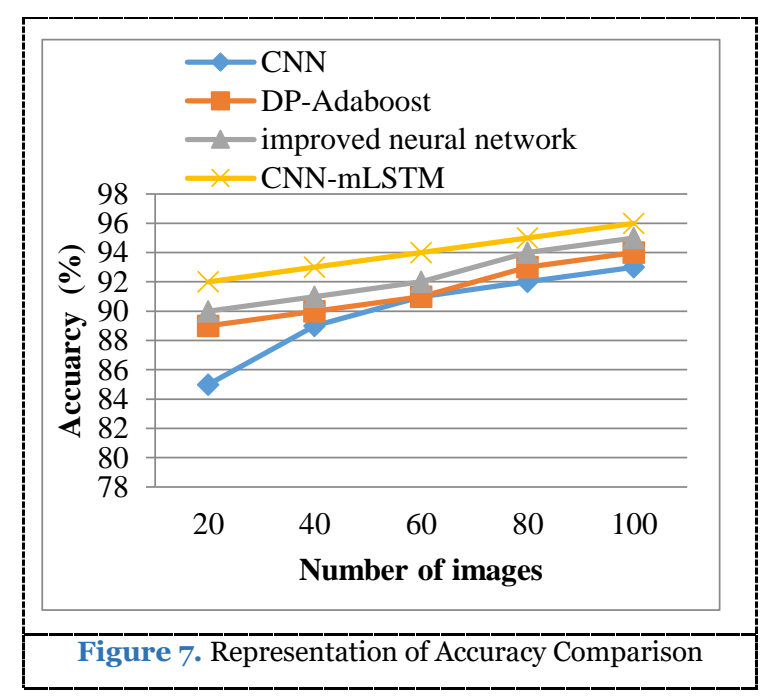

This is due to the automatic extraction of the features using CNN in the CNN-mLSTM classification algorithm, which increases the classification precision resulting in Brain tumour. The numerical results of Accuracy Comparison is shown in Table 4.

Table 4. The numerical results of Accuracy Comparison

\begin{tabular}{ccccc}
\hline $\begin{array}{c}\text { No.of } \\
\text { images }\end{array}$ & CNN & $\begin{array}{c}\text { DP- } \\
\text { Adaboost }\end{array}$ & $\begin{array}{c}\text { improved } \\
\text { neural } \\
\text { network }\end{array}$ & $\begin{array}{c}\text { CNN- } \\
\text { mLSTM }\end{array}$ \\
\hline 20 & 85 & 89 & 90 & 92 \\
\hline 40 & 89 & 90 & 91 & 93 \\
\hline 60 & 91 & 91 & 92 & 94 \\
\hline 80 & 92 & 93 & 94 & 95 \\
\hline 100 & 93 & 94 & 95 & 96
\end{tabular}

\section{CONCLUSION AND FUTURE} WORK

In this work, face recognition is done using proposed CNN-mLSTM. The overall performances were obtained using the different number of training images and test images. Initially, after the pre-processing the Retinex-based adaptive filter is applied to enhance the face images. The convolutional neural networks achieve the best results of feature extraction so far. This work proposed to use a LSTM network and compare its performance with a standard MLP network for face classification problems. The mLSTM network presented for face recognition which can attain better performance in terms of correct classification rates in all the three proposed face classification tasks, showing that it is a powerful tool in face recognition applications, even if dealing with a reduced training set. The CNN-mLSTM achieves a much better recognition performance than the conventional schemes such as CNN, DP-Adaboost and improved neural network. The proposed system can be extended by using the classifiers like deep learning with the various optimization schemes like, genetic algorithm etc.

\section{REFERENCES}

[1]. Ding, C., \& Tao, D. (2016). A comprehensive survey on poseinvariant face recognition. ACM Transactions on intelligent systems and technology (TIST), 7(3), 1-42.

[2]. Lei, Z., Yi, D., \& Li, S. Z. (2015). Learning stacked image descriptor for face recognition. IEEE Transactions on Circuits and Systems for Video Technology, 26(9), 16851696.

[3]. Zhang, Z., Luo, P., Loy, C. C., \& Tang, X. (2015). Learning deep representation for face alignment with auxiliary attributes. IEEE transactions on pattern analysis and machine intelligence, $38(5)$, 918-930.

[4]. Bharati, A., Singh, R., Vatsa, M., \& Bowyer, K. W. (2016). Detecting facial retouching using supervised deep learning. IEEE Transactions on Information Forensics and Security, 11(9), 1903-1913.

[5]. Ding, C., \& Tao, D. (2016). A comprehensive survey on poseinvariant face recognition. ACM Transactions on intelligent systems and technology (TIST), 7(3), 1-42.

[6]. Zhang, K., Zhang, Z., Li, Z., \& Qiao, Y. (2016). Joint face detection and alignment using multitask cascaded convolutional networks. IEEE Signal Processing Letters, 23(10), 1499-1503.

[7]. Wu, H., Zhang, K., \& Tian, G. (2018). Simultaneous face detection and pose estimation using convolutional neural network cascade. IEEE Access, 6, 49563-49575.

[8]. Yadav, S., \& Nain, N. (2016). A novel approach for face detection using hybrid skin color model. Journal of Reliable Intelligent Environments, 2(3), 145-158.

[9]. Zheng, Y. Y., \& Yao, J. (2015). Multi-angle face detection based on DP-Adaboost. International Journal of Automation and Computing, 12(4), 421-431.

[10]. Luo, D., Wen, G., Li, D., Hu, Y., \& Huan, E. (2018). Deeplearning-based face detection using iterative bounding-box regression. Multimedia Tools and Applications, 77(19), 24663-24680.

[11]. Yoganand, A. V., \& Kavida, A. C. (2018). Face detection approach from video with the aid of KPCM and improved neural network classifier. Multimedia Tools and Applications, $77(24), 31763-31785$. 